\title{
Les canaux ioniques dans l'anoxie cérébrale : cibles possibles d'agents protecteurs
}

L'anoxie cérébrale peut être aisément étudiée dans le modèle expérimental des tranches de tissu hippocampique incubées dans des solutions contenant ou non de l'oxygène. L'anoxie bloque la transmission synaptique et entraîne des modifications séquentielles de l'excitabilité cellulaire. Les phénomènes en cause dans ces perturbations semblent être la baisse de l'ATP intracellulaire, l'augmentation $\mathrm{du} \mathrm{Ca}^{+}+$cytoplasmique et l'importante libération excitotoxique de glutamate qui va, notamment, activer les canaux NMDA (N-méthylD-aspartate), aggravant les lésions cellulaires du fait d'un flux entrant massif de $\mathrm{Ca}^{+}{ }^{+}$. Des médicaments bloquant les canaux calciques voltage-dépendants et les canaux NMDA vont donc avoir un effet protecteur potentiel contre l'anoxie. L'ouverture des canaux $\mathbf{K}^{+}$ dépendants de l'ATP, créant une hyperpolarisation cellulaire et donc une hypoexcitabilité, a également un rôle protecteur qui pourrait être mis à profit par l'utilisation de modulateurs spécifiques de ces canaux.

\section{Yehezkel Ben-Ari}

e système nerveux central est extrêmement vulnérable à l'interruption même brève de son oxygénation. En cas d'anoxie complète, on observe rapidement une perte de la connaissance [1] ct une disparition de l'activité EEG. Ces deux phénomènes traduisent l'interruption de la transmission synaptique dont nous envisagerons le mécanisme assez complexe. Lorsque l'anoxie se répète ou se prolonge, on observe des lésions touchant prioritairement des neurones, premières cellules cérébrales à souffrir, avant la glie et les vais- seaux. L'anoxie cérébrale, liée dans la majorité des cas à des désordres circulatoires, représente un problème de santé majeur, particulièrement chez le sujet âgé. Que les lésions soient focales ou plus diffuses, elles peuvent entraîner des déficits moteurs ou cognitifs très invalidants. Ainsi, il est bien établi que dans les démences des sujets âgés, à côté de la maladie d'Alzheimer, il faut faire une place aux lésions ischémiques multifocales du SNC [2].

Les conséquences des lésions vasculaires du SNC (système nerveux central) ne sont cependant pas toutes 


\section{RÉFÉRENCES}

1. Himwich HE. Brain metabolism and cerebrall disorders. Baltimore: Williams and Wilkins, 1951.

2. Delay J, Brion S. “Les Démences Tardives ». Paris : Masson, 1962.

3. Watkins JC, Olverman HJ. Antagonists and antagonists for excitation acids receptors. Trends Neurosci $1987 ; 10: 265-72$.

4. Neuman RS, Ben-Ari Y, Cho M, Cherubini E. Blockade of excitatory synaptic transmission by 6-cyano-7-nitroquinoxaline-2,3-dione (CNQX) in the hippocampus in vitro. Neurosci Letts 1988 ; 92 : 64-8.

5. Pulsinelli WA, Bierley JB, Plum F. Temporal profile of neuronal damage in a model of transient forebrain ischemia. Ann Neurol 1982; 11 : 491-4.

6. Kirino T. Delayed neuronal death in the gerbil hippocampus following ischemia. Brain Res 1982 ; 239 : 57-69.

7. Zola-Morgan S, Squire Larry R, Amara David G. Human Amnesia and the Medial Temporal Region: enduring memory impairment following a bilateral lesion limited to field CA1 of the hippocampus. $J \mathrm{Neu}$ rosci $1986 ; 6: 2950-67$.

8. Choi DW. Calcium-mediated neurotoxicity : relationship to specific channel types and role in ischemic damage. Trends Neurosci $1988 ; 11$ : 465-9.

9. Benveniste $H$, Drejer J, Schousboe N, Diemer NH. Elevation of the extracellular concentration of glutamate and aspartate in rat hippocampus during transient cerebral ischemia monitored by intracerebral microdialysis. J Neurochem 1984; 43 : 1369-74.

10. Cherubini E, Ben-Ari Y, Krnjevic K. Anoxia produces smaller changes in synaptic transmission membrane potential and input resistance in immature rat hippocampus. $J$ Neurophysiol 1989a ; 62 : 882-95.

11. Krnjevic K, Cherubini E, Ben-Ari Y. Anoxia on slow inward current of immature hippocampal neurons. $J$ Neurophysiol 1989 ; 62 : 896-906.

12. Hansen AJ. Effect of anoxia on ion distribution in the brain. Physiol Rev 1985 ; $65: 101-48$.

13. Fujiwara $N$, Higashi $H$, Shimoji $K$, Yoshimura M. Effects of hypoxia on rat hippocampal neurones in vitro. $J$ Physiol Lond 1987 ; 384 : 131-51. inéluctables. Spontanément, l'évolution des lésions est partiellement réversible sur les plans clinique et anatomique. Le phénomène est lié à la structure de l'infarctus cérébral : celui-ci comprend toujours une zone centrale, où les cellules nerveuses sont définitivement détruites, et une zone périphérique, dite de pénombre, où les lésions sont partiellement réversibles. Cette régression spontanée partielle peut être améliorée par un certain nombre d'agents pharmacologiques, du moins pendant une période de quelques heures propice à l'activité anti-anoxique de certaines molécules.

Dans cette revue, les effets de l'anoxie sur la transmission synaptique et l'excitabilité cellulaire seront décrits et les stratégies de développement d'agents permettant de réduire les lésions anoxiques seront discutées. Auparavant, nous rappellerons brièvement l'organisation de la formation hippocampique ; c'est, en effet, sur cette structure cérébrale particulièrement vulnérable à l'anoxie que la plupart des études expérimentales ont été effectuées.

\section{La formation hippocampique : un modèle pour l'étude de l'anoxie}

L'hippocampe est une structure corticale simplifiée, organisée de façon laminaire. Elle est composée schématiquement de trois types de neurones : les grains, les cellules pyramidales et les interneurones. La couche des grains et celle des cellules pyramidales sont facilement identifiables dans une coupe histologique grâce à la forme caractéristique de deux croissants qui s'emboîtent (figure 1A). Les interneurones sont, en revanche, principalement dispersés à l'extérieur des couches principales, c'est-à-dire le long des dendrites, des grains et des pyramides. La propagation de l'influx nerveux dans la formation hippocampique se fait le long d'un réseau trisynaptique composé successivement : (1) du faisceau perforant, principale afférence extrinsèque de l'hippocampe qui provient du cortex entorhinal et qui innerve les dendrites apicaux des grains; (2) des fibres moussues, axones des grains qui innervent la partie proximale des dendrites apicaux des cellules pyramidales géantes de la région inférieure (CA3); (3) des collatérales anoxales de Schaffer, provenant des neurones pyramidaux de CA3 et qui innervent le dendrite apical des neurones pyramidaux de la région supérieure (CA1).

Le médiateur de ces trois synapses excitatrices est vraisemblablement l'acide glutamique, qui peut agir sur au moins deux types de récepteurs : les récepteurs canaux quisqualatekainate perméables aux cations monovalents $\left(\mathrm{Na}^{+}, \mathrm{K}^{+}\right)$et les récepteurs canaux NMDA (Nméthyl-D-aspartate) également perméables au $\mathrm{Ca}++[3]$. Le canal du récepteur NMDA est soumis au repos à un blocage, dépendant du voltage par le $\mathrm{Mg}^{+}+$présent dans le milieu extracellulaire. Une forte dépolarisation est nécessaire pour lever ce blocage et permettre l'activation du canal. Dans des conditions de faible stimulation, le récepteur canal NMDA intervient peu dans la transmission synaptique, et des antagonistes sélectifs des récepteurs non NMDA bloquent entièrement les potentiels synaptiques excitateurs des différentes connexions hippocampiques [4]. En revanche, dans des conditions de forte stimulation (notamment pendant l'anoxie), la dépolarisation va lever le blocage du canal -NMDA par le $\mathrm{Mg}^{+}+$et induire par conséquent un flux entrant de $\mathrm{Ca}++$ dans la cellule, phénomène qui va jouer un rôle crucial dans les séquelles lésionnelles (voir plus bas). L'hippocampe est particulièrement vulnérable à l'hypoxie. Il existe un gradient de vulnérabilité des cellules hippocampiques à l'anoxie. La région de CA1 (ou le secteur de Sommer chez l'homme) est très vulnérable, suivie de CA3, alors que les grains apparaissent plus résistants. Ainsi, chez plusieurs espèces animales, une occlusion de brève durée des carotides provoque - dans un délai de 2 à 3 jours - une lésion complète et sélective des cellules pyramidales de CA1 sans affecter les autres neurones de l'hippocampe ou ceux d'autres structures cérébrales $[5,6]$. Une même vulnérabilité du secteur de Sommer a été également observée chez l'homme. Ainsi, on a décrit, dans la littérature, des accidents vas- 
culaires péri-anesthésiques ayant entraîné une perte de la mémoire antérograde ; l'examen post-mortem, effectué sur ces patients quelques années plus tard, révélait une destruction sélective du secteur de Sommer [7]. Ces données confirment à la fois la vulnérabilité de CA1 à l'anoxie et le rôle de cette région dans la fonction mnésique.

De nombreuses données expérimentales $[8,9]$ suggèrent que la lésion anoxique des cellules hippocampiques est due à une libération excitotoxique de glutamate induite par le déséquilibre ionique que cause une anoxie prolongée. La dépolarisation massive des neurones par le glutamate va engendrer un flux entrant de calcium dans la cellule par l'intermédiaire aussi bien des canaux calciques dépendant du voltage que des canaux associés au récepteur NMDA. L'augmentation $\mathrm{du} \mathrm{Ca}++$ intracellulaire qui en résulte va induire la mort cellulaire en activant des protéases dépendantes du $\mathrm{Ca}++$ qui vont détruire les protéines constituantes du cytosquelette; ou encore en activant certaines phospholipases, qui peuvent dégrader les lipides membranaires et provoquer la formation de radicaux libres.

\section{Effets électrophysiologiques de I'anoxie}

Une étude électrophysiologique est indispensable afin de déterminer les effets immédiats de l'anoxie sur la décharge cellulaire et la cascade de mécanismes pouvant induire, dans une étape ultérieure, la mort neuronale. Il est difficile d'effectuer ce type d'études dans de bonnes conditions sur l'animal entier. En revanche, la préparation de tranches d'hippocampe maintenues en survie artificielle permet de mieux contrôler les conditions expérimentales (milieu ionique, concentration d'agents pharmacologiques, température locale) tout en évitant les effets systémiques de l'anoxie. Sur ce type de préparation, on peut effectuer des enregistrements durables (plusieurs heures) des réponses électrophysiologiques évoquées par l'activation de fibres afférentes au niveau d'une cellule (enregistrement intracellulaire) ou d'une population homogène de cellules (enregistrement extracellulaire). $m / s n^{\circ} 1$, rol. 7 , janvier 91
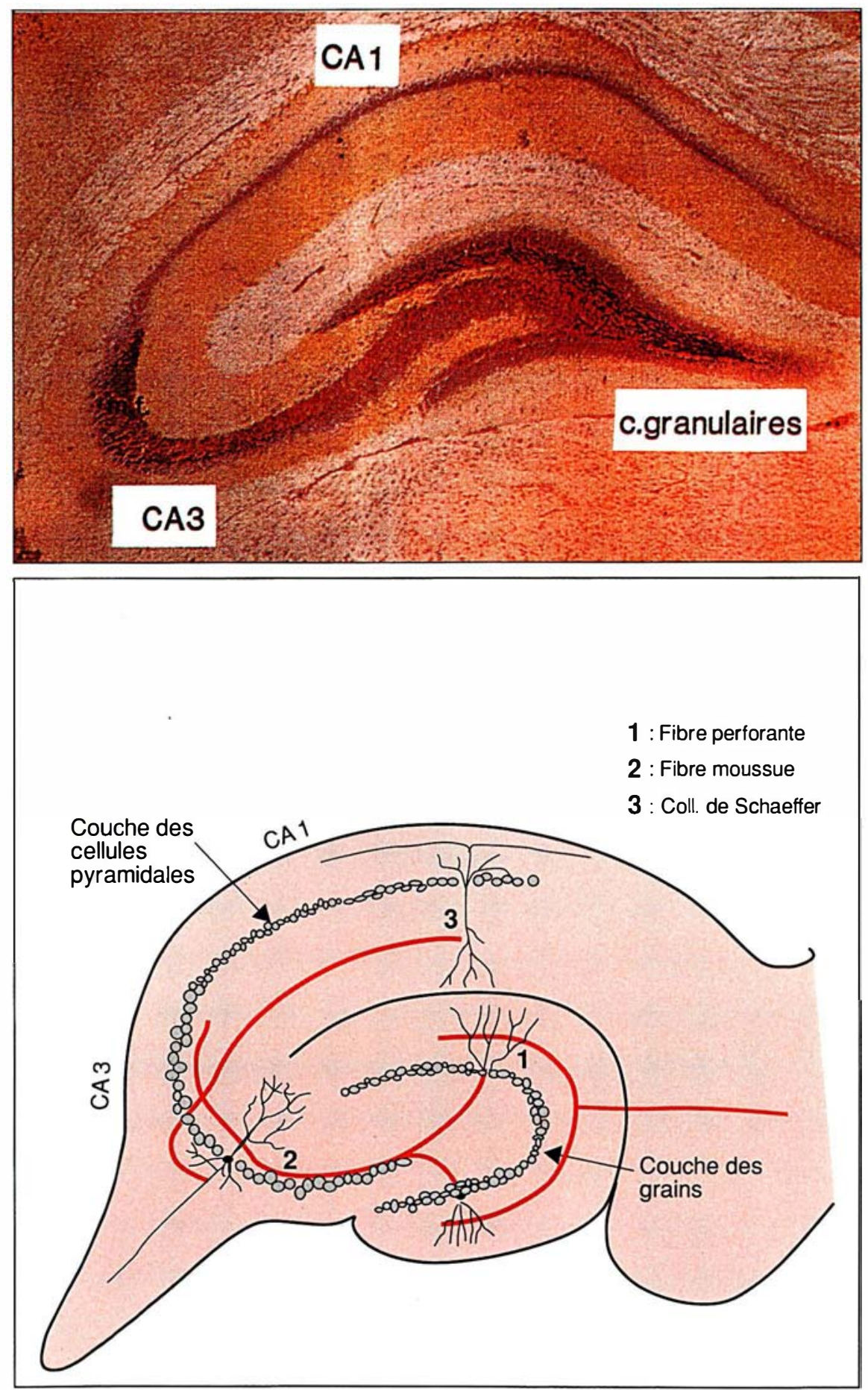

Figure 1. Schéma de I'hippocampe et coupe histologique. CA1 : région supérieure de l'hippocampe. CA3 : région inférieure de l'hippocampe.

L'anoxie, produite en perfusant la tranche avec un milieu dépourvu d'oxygène, bloque la transmission synaptique et produit une cascade d'effets sur l'excitabilité cellulaire. Ces effets sont réversibles tant que la durée de l'anoxie n'excède pas quel- ques minutes; au-delà de cette période, on observe une dépolarisation brutale de plusieurs dizaines de millivolts du potentiel de repos, et il n'y a pas de récupération des propriétés électriques au retour en milieu oxygéné [10-14]. 


\section{RÉFÉRENCES}

14. Hansen AJ, Houngaard J, Jahnsen $\mathrm{H}$. Anoxia increases potassium conductance in hippocampal nerve cells. Acta Physiol Scand 1982 ; 115 : 301-10

15. Ben-Ari Y. Galanine and glibenclamide modulate the anoxic release of glutamate in rat CA3 hippocampal neurons. Eur J Neurosci $1990 ; 2$ : 62-8.

16. Krnjevic K, Leblond J. Anoxia reversibly suppresses neuronal Ca-currents in rat hippocampal slices. Can $J$ Physiol Pharmacol 1989 ; 65 : 2157-61.

17. Lipton P. and Whittingham TC. Reduced ATP concentration as a basis for synaptic transmission failure during hypoxia in the in-vitro guinea pig hippocampus. $J$ Physiol 1982 ; 325 : 51-65.

18. Eckert R, Chad JE. Inactivation of $\mathrm{Ca}$ channels. Prog Biophys Mol Biol 1984 44: 215-76.

19. Byerly L, Yazejian B. Intracellular factors for the maintenance of calcium currents in perfused neurones from the snail, Lymnae stagnalis. J Physiol Lond 1986; $370: 631-50$.

20. Sanchez-Prieto J, Gonzalez P. Occurence of a large $\mathrm{Ca}^{2}+$ independant release of glutamate during anoxi in isolated terminals (synaptosomes). J Neurochem 1988 ; $50: 1322-4$

21. Ikeda $M$, Nakazawa $T$, Abe $K$, Kameto T, Yamatsu ?. Extracellular accumulation of glutamate in the hippocampus induced by ischemia is not calcium dependant in vivo and in vitro evidence. Neurosci Letl 1989 ; 26 : 202-6.

22. Rader RK, Lanthorn ?. Experimental ischemia induces a persistent depolarization blocked by decreased Calcium and NMDA antagonist. Neurosci Lett 1989 ; 99 : 125-30.

23. Lazdunski M. Les canaux $\mathrm{K}^{+}$potassiques sensibles à l'ATP ou les suites imprévues de l'étude des sulfamides hypoglycemiants. médecine/sciences $1990 ; 6: 279-85$.

24. Ben Ari Y, Krnjevic K, Crepel V. Activators of ATP ${ }^{+}$sensitive $\mathrm{K}^{+}$channels reduce anoxic hyperpolarization in CA3 hippocampai neurons. Neurosciences 1990 ; 37 :

Nous résumerons brièvement les principaux effets de brèves périodes d'anoxie avant de proposer un schéma des mécanismes sous-jacents.

\section{- Effets de l'anoxie sur la trans-} mission synaptique

L'anoxie bloque la transmission synaptique. En effet, les potentiels postsynaptiques excitateurs (PPSE) évoqués par stimulation électrique des axones sont bloqués très rapidement par un milieu dépourvu d'oxygène $[10,11,13-15]$. Il y a une bonne corrélation sur le plan régional entre le délai de blocage de la transmission et la vulnérabilité aux séquelles lésionnelles de l'anoxie. Ainsi, dans des tranches d'hippocampe adulte, 2 minutes d'anoxie suffisent à bloquer complètement la réponse synaptique au niveau de CA1 (la région vulnérable, figure 2), alors que 5-10 minutes sont nécessaires pour les fibres moussues de la région de CA3 [10, 11]. De même, avec K. Krnjevic et E. Cherubini [11], nous avons observé qu'il fallait 8-10 minutes d'anoxie pour bloquer la transmission synaptique au niveau de CA1 dans des tranches de rat âgé de 1 à 4 jours. Cette observation est en accord avec les nombreuses données expérimentales et cliniques montrant une plus grande résistance du tissu immature à l'anoxie [12].

Concernant les mécanismes de ces effets, il est clair que l'interruption par l'anoxie de la transmission synaptique n'est pas due à un blo-

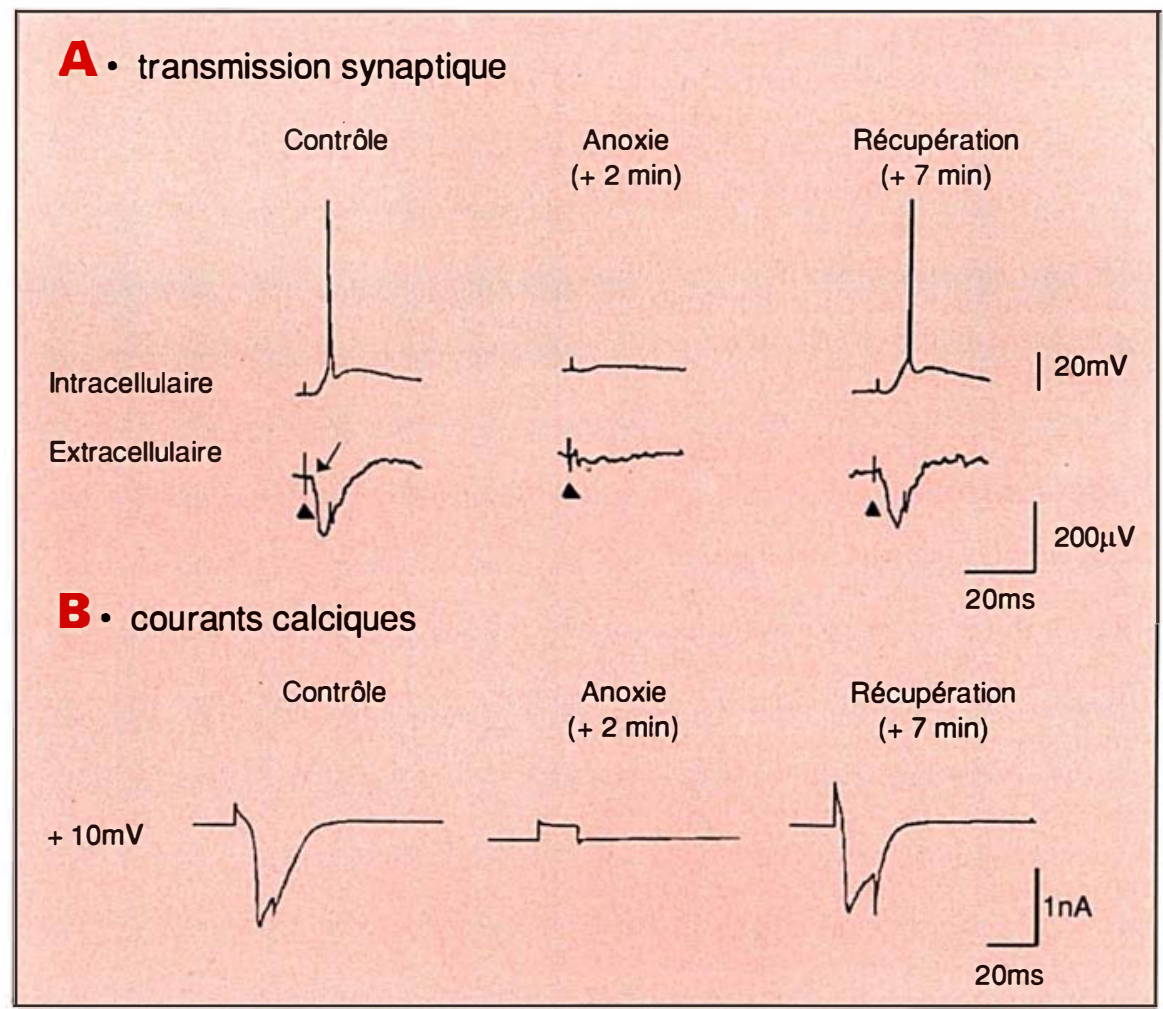

Figure 2. L'anoxie bloque la transmission synaptique et les courants calciques de la région de CA1. A : la réponse synaptique évoquée par la stimulation des collatérales de Schaeffer au niveau de CA 1 est abolie 2 minutes après la perfusion de la tranche par un milieu dépourvu d'oxygène. Le potentiel précoce qui correspond à la propagation de l'influx nerveux le long des axones présynaptiques n'est pas réduit par l'anoxie. B : les courants calciques évoqués par une impulsion dépolarisante dans une cellule pyramidale de CA1 sont abolis par l'anoxie. Ces courants ont été enregistrés par la méthode dite de voltage imposé à une seule électrode; les courants ioniques transmembranaires sont mesurés après avoir imposé un voltage à la cellule enregistrée. Les tranches sont perfusées avec un milieu contenant des bloquants des canaux sodiques et potassiques afin de permettre l'activation sélective des courants calciques relativement purs. 


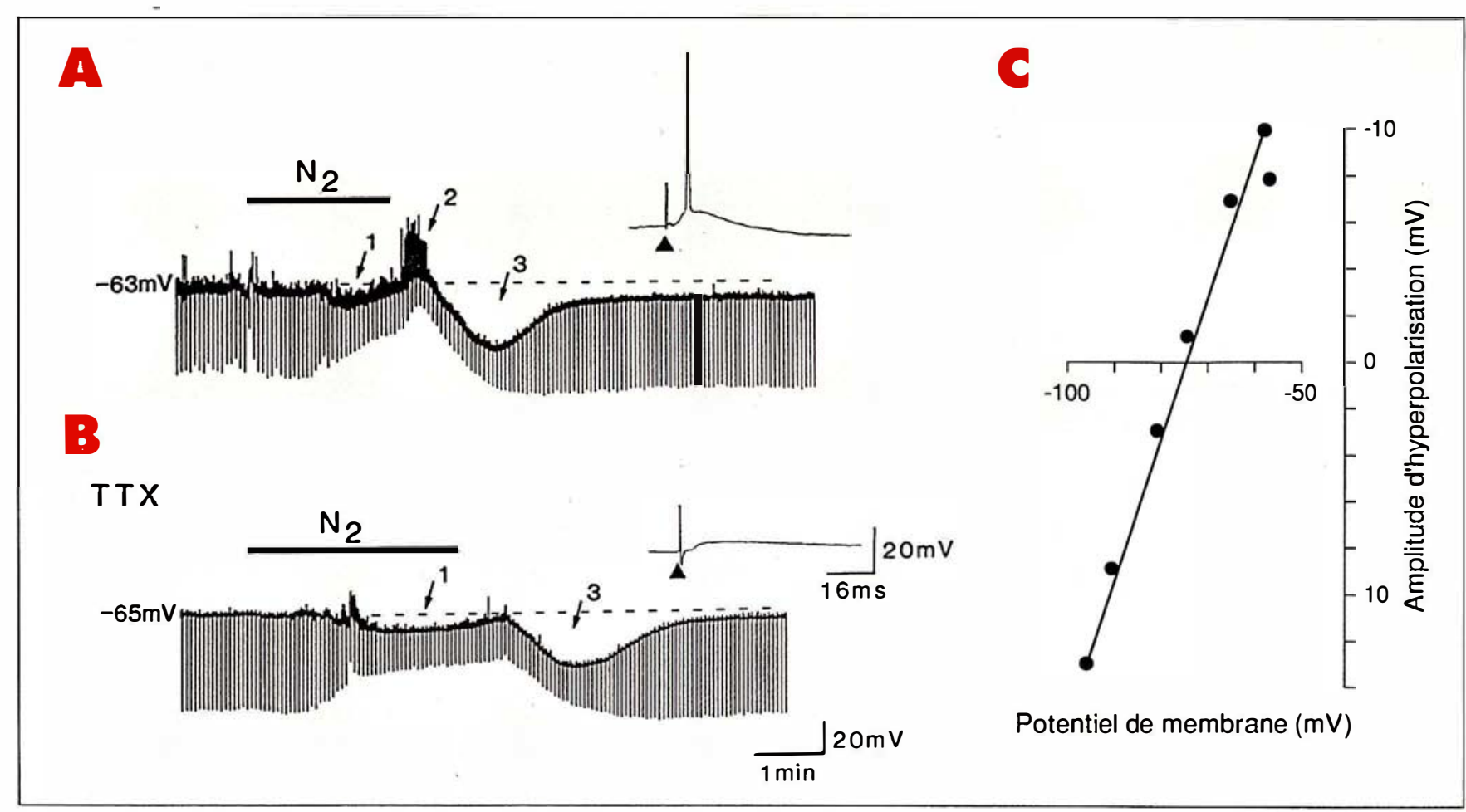

Figure 3. Effets de l'anoxie sur l'excitabilité d'une cellule pyramidale de la région de CA3. A : noter la séquence hyperpolarisation (1), dépolarisation (2) et hyperpolarisation post-anoxique (3). En présence de tétrodotoxine pour bloquer des canaux sodiques (B) la dépolarisation (phase 2) est bloquée, indiquant qu'elle est d'origine synaptique. $C$ : inversion de l'hyperpolarisation anoxique. Les barres horizontales représentent des impulsions de courant hyperpolarisants destinées à mesurer la résistance cellulaire pendant les différentes phases de l'anoxie. Noter la réduction de la résistance (baisse de l'amplitude des barres horizontales) pendant l'anoxie.

cage de la propagation de l'influx nerveux le long des éléments présynaptiques (figure $2 A$ ). Cela est d'ailleurs en accord avec de nombreuses expériences qui montrent une résistance relative de l'activité électrique de l'axone en situation d'anoxie. De plus, l'interruption ne semble pas résulter d'une incapacité de l'élément postsynaptique à engendrer des potentiels d'action, puisque la stimulation directe de la cellule pyramidale par un courant dépolarisant peut engendrer un tel potentiel d'action bien après que la transmission a été abolie. L'interruption de la transmission synaptique est par conséquent due à un blocage de la transmission résultant probablement d'une inacti$m / s n^{\circ} 1$, vol. 7, janvier 91 vation des courants calciques nécessaires à la libération de transmetteur (voir plus bas).

- Effets de l'anoxie sur l'excitabilité cellulaire

Une brève période d'anoxie produit d'abord, régulièrement, une hyperpolarisation ; celle-ci est souvent suivie d'une dépolarisation (figure $3 A$ ). Le retour en milieu oxygéné s'accompagne d'une seconde hyperpolarisation dite postanoxique (HPA). L'hyperpolarisation anoxique (HA) s'accompagne d'une baisse de la résistance membranaire (figure $3 \mathrm{~A}$ ). Elle est due à l'activation par l'anoxie d'un canal perméable au $\mathrm{K}+$; la sortie de $\mathrm{K}+$ (en fonction du gradient de concentration) va se traduire par une hyperpolarisation. En effet, si l'on impose à la cellule un potentiel voisin du potentiel d'équilibre de $\mathrm{K}^{+}$, l'hyperpolarisation anoxique s'annule (figure $3 C$ et $[12,13,15])$. Le potentiel d'équilibre du potassium, $\mathrm{E}_{\mathrm{K}}$, est celui où les différentes forces électriques et thermiques s'équilibrent et où les mouvements d'ions $\mathrm{K}+$ à travers le canal (dans le sens entrant et sortant) s'annulent. L'ouverture des canaux $\mathrm{K}+$ constitue un mécanisme d'inhibition particulièrement efficace dans le SNC comme dans certaines structures périphériques, tels les muscles lisses, où l'ouverture de canaux $\mathrm{K}^{+}$ se traduit par une relaxation. 


\section{RÉFÉRENCES}

25. Thuringer D and Escande D. Apparent competition between ATP and the potassium channel opener RP 49356 on ATP sensitive $\mathrm{K}^{+}$channels of cardiac myocytes. Mol Pharmacol 1989 ; 36 : 897-902.

26. Facci L, Milani D, Leon A, Shappee SD. Monosialaganglioside GM1 protects against anoxic and kainic acid induced neuronal death in vitro. 12th ISN Biennal Meeting 89, International Society for Neurochemisty. Algarve, Portugal, April 23/28, 1989.

27. Acosta D, Kass IS, Cotrell JE. Effect of and Tocopherol and free radicals on anoxic damage in the rat hippocampal slice. Exp Neurol 1987 ; 98 : 607-14.

28. Kass IS, Cotrell JE, Chambers G. Magnesium and cobalt but not nimodipine protect neurons against anoxic damage in the rat hippocampal slice. Anasthesiology $1988 ; 69: 710-5$

29. Steen PA, Newberg LA, Milde JH, Michen Felder JD. Nimodipine improves cerebral blood flow and neurologic recory after complete cerebral ischemia in the dog $J$ Cerebr Blood Flow Metab 1983 ; 3 : 38-43.

30. Simon RP, Swan JH, Griffiths T, Meldrum BS. Blockade of N-methyl-D- aspartate receptors may protect against ischemic damages in the brain. Science 1984; 226 : 850-2.

31. Dunwiddie TV. The physiological role of adenosine in the central Nervous System. Int Rev Neurobiol 1985 ; 27 : 63-139.

32. Fowler JC. Adenosine antagonists alter the synaptic response to in vitro ischemia in the rat hippocampus. Brain Res $1990 ; 509$ : $331 \cdot 4$

33. Miller R. Glucose regulated $\mathrm{K}+$ channels are sweet news for neurobiologists. Trends Neurosci 1990 ; 13 : 197-9.

34. Mourre C, Ben-Ari Y, Bernadi H, Fosset M, Lazdunski M. Antidiabetic sulfonylureas : localization of binding sites in the brain and effects on the hyperpolarization induced by anoxia in hippocampal slices. Brain Res 1989 ; 486 : 159-64.

35. Ashcroft FM. Adenosine 5'-triphophatesensitive potassium channels. Ann Rev Neurosci $1988 ; 11$ : 97-118.

36. Ashford MLJ, Sturgess NC, Trout NJ, Garner NJ, Hales CN. Adenosine-5'triphosphate- sensitive ion channels in neonatal rat cultured central neurones. Pflüg Arch 1988 ; 412 : 297-304.

37. Noma A. A TP regulated $\mathrm{K}+$ channels
L'hyperpolarisation a un rôle homéostatique, elle va empêcher une excitation accrue (et donc une consommation énergétique excessive) à un moment ou les réserves métaboliques sont défaillantes. Des données récentes suggèrent que parmi les nombreux types de canaux $\mathrm{K}+$, $\mathrm{HA}$ est due en particulier à l'ouverture de canaux dépendants du calcium [14-16]. Ces canaux ont la particularité d'être activés par une augmentation $\mathrm{du} \mathrm{Ca}^{+}+$intracellulaire $\mathrm{du}$ type de celle que produit l'anoxie (voir plus loin).

La dépolarisation anoxique (DA) est due à une libération accrue de médiateurs, notamment de glutamate [15]. DA est en effet bloquée par des antagonistes des récepteurs glutamatergiques ; elle est également bloquée par la tétrodotoxine qui bloque les courants $\mathrm{Na}^{+}$et par conséquent la propagation de l'influx nerveux et les réponses synaptiques évoquées par une stimulation électrique des axones présynaptiques (figure $3 A$ ). Par conséquent, contrairement à HA, la dépolarisation anoxique est d'origine présynaptique.

L'hyperpolarisation postanoxique (HPA) est due à la réactivation par la réoxygénation au niveau de l'élément post-synaptique de la pompe $\mathrm{Na}+-\mathrm{K}+$ électrogénase [13-16]. Cette pompe a pour rôle de maintenir l'équilibre sodico-potassique en faisant entrer du $\mathrm{K}+$ et sortir du $\mathrm{Na}^{+}$. Son activation accélérée après l'anoxie va permettre de résorber rapidement l'accumulation extracellulaire de $\mathrm{K}+$ et intracellulaire de $\mathrm{Na}^{+}$. En revanche, si l'anoxie est prolongée, on n'observe plus de HPA et la viabilité de la tranche est rapidement compromise.

\section{Mécanismes cellulaires de l'anoxie}

Un des tout premiers effets de l'anoxie est de réduire les concentrations d'ATP intracellulaires par interruption de la phosphorylation oxydative. La mesure des concentrations d'ATP dans des préparations membranaires ne révèle cependant pas d'emblée une baisse massive après l'anoxie ; on peut suggérer qu'une distribution hétérogène de l'ATP masque la baisse importante au niveau de sites sensibles. Plusieurs données expérimentales suggèrent que la baisse d'ATP intracellulaire est en fait à l'origine du blocage par l'anoxie de la transmission synaptique [17]. Une des conséquences de la baisse d'ATP va être d'augmenter les concentrations de $\mathrm{Ca}++$ intracellulaire du fait de l'arrêt de pompes et d'échangeurs qui contrôlent de façon très stricte le $\mathrm{Ca}++$ libre dans le milieu intracellulaire à des taux très bas (de l'ordre de $10^{-i}-10^{-8} \mathrm{M}$ ) [8]. La baisse d'ATP et l'augmentation de $\mathrm{Ca}++$ constituent la cause des effets pré- et post-synaptiques de brèves périodes d'anoxie.

Ainsi, au niveau des terminaisons nerveuses, l'augmentation de $\mathrm{Ca}++$ et la baisse d'ATP produisent un blocage réversible de la transmission synaptique évoquée. Il est probable que cela est dû à l'inactivation des courants " $\mathrm{Ca}++$ dépendants " du voltage responsables du flux entrant de calcium nécessaire à la libération évoquée de médiateur. Du fait de la taille des terminaisons, il n'est pas possible d'y mesurer l'amplitude des courants calciques (et les flux de $\left.\mathrm{Ca}^{+}{ }^{+}\right)$; on peut cependant étudier les effets de l'anoxie sur les courants calciques " voltage-dépendants " enregistrés au niveau du corps cellulaire. Comme le montre la figure $2 B$, l'anoxie bloque effectivement les courants calciques ; de plus, ce blocage est plus rapide au niveau de CA1 que de CA3 et dans l'hippocampe adulte que dans l'organe immature [11]. Il y a par conséquent une excellente corrélation entre le blocage des courants calciques et l'interruption de la transmission synaptique (voir également [16]). Les mécanismes de l'inactivation des courants calciques n'ont pas été entièrement élucidés ; elle pourrait être directement due soit à l'augmentation du $\mathrm{Ca}^{+}+$, soit à l'accumulation d'un facteur intracellulaire qui va inactiver ces courants, soit encore à une réduction - du fait de la baisse d'ATP - de la phosphorylation des canaux calciques nécessaire à leur fonctionnement [18, 19].

$\mathrm{Au}$ niveau de l'élément postsynaptique, l'augmentation de $\mathrm{Ca}+{ }^{+}$induit l'activation du courant $\mathrm{K}+$ dépendant du $\mathrm{Ca}++$, avec comme conséquences, une hyperpo- 
larisation anoxique et une réduction de l'excitabilité neuronale; il s'agit d'un mécanisme protecteur destiné à empêcher une hyperexcitabilité dans une situation de stress métabolique. Parallèlement à ses effets sur la transmission synaptique (évoquée), l'anoxie affecte également la libération spontanée de médiateur. On observe en effet, pendant des anoxies relativement brèves, une dépolarisation due à une libération de transmetteurs - notamment de glutamate. Des données biochimiques [20, 21] et physiologiques [22] suggèrent que cette libération, qui est relativement insensible au $\mathrm{Ca}++$ extracellulaire, pourrait provenir d'un autre compartiment de médiateur. Quel que soit le mécanisme exact de cette libération, il est clair que si l'anoxie est plus sévère (par exemple, une anoxie plus durable accompagnée d'une hypoglycémie), on observe une dépolarisation plus ample associée à une perte irréversible de la transmission synaptique et une nécrose cellulaire 24 à 48 heures plus tard. D'où l'importance des données récentes qui suggèrent que les canaux $\mathrm{K}+$-dépendants de l'ATP (voir encart et [15]), qui jouent un rôle mineur au niveau postsynaptique dans la réponse anoxique, modulent la libération spontanée de glutamate et par conséquent la dépolarisation anoxique. En effet, dans un travail récent, nous avons pu montrer que des agents qui bloquent sélectivement les canaux $\mathrm{K}+$ dépendants de l'ATP comme la glibenclamide, augmentent la dépolarisation anoxique des cellules hippocampiques. En revanche, l'hormone hyperglycémiante galanine, qui active ces canaux [23], bloque la dépolarisation anoxique [15]. Le diazoxyde, un activateur sélectif de ces canaux, a le même effet [24]. Il est intéressant de noter que ces agents pharmacologiques ont relativement peu d'effets en milieu oxygéné sur la décharge neuronale et n'affectent pas l'excitabilité cellulaire une fois que la transmission synaptique a été bloquée par la tétrodoxine. C'est par conséquent au niveau des terminaisons nerveuses qu'il faut situer leurs actions. Je propose que l'anoxie produit une désinhibition partielle des canaux $\mathrm{K}+$ dépendants de l'ATP $\mathrm{m} / \mathrm{s} n^{\circ} 1$, vol. 7 , janvier 91 par l'intermédiaire d'une baisse de l'ATP intracellulaire ; cet effet, cependant, ne suffit pas à compenser les autres actions de l'anoxie qui tendent à augmenter la libération des médiateurs. Des hormones ou des drogues qui facilitent l'ouverture de ces canaux, notamment en déplaçant l'ATP de son site de blocage du canal [25], vont produire une hyperpolarisation au niveau des terminaisons, et par conséquent une réduction de la libération de glutamate.

\section{Stratégies de développement de molécules pourvues d'effet protecteur}

Au vu des données précédentes, nous voyons qu'il est théoriquement possible d'intervenir à plusieurs niveaux afin de réduire les effets de l'anoxie cérébrale. De très nombreuses molécules réduisent effectivement l'étendue des lésions anoxiques in vivo (ischémie globale ou focale) chez le rat ou la gerbille ou in vitro sur des cultures de cellules hippocampiques ou sur des tranches d'hippocampe. Ces effets protecteurs correspondent à autant de sites d'actions possibles ; ainsi des données expérimentales intéressantes ont été obtenues avec les gangliosides, qui renforcent les constituants membranaires [26], ou encore avec le tocophérol, qui empêche la formation de radicaux libres [27]. Cependant, l'effort principal de l'industrie pharmaceutique porte à l'heure actuelle sur le développement de molécules susceptibles d'agir sur les sites suivants :

- Les canaux calciques [28, 29]

En empêchant l'activation des canaux calciques "voltage-dépendants ", et donc en partie l'accumulation de $\mathrm{Ca}$ intracellulaire, des agents comme la nifédipine peuvent réduire l'excitotoxicité anoxique. Des données très contradictoires ont cependant été obtenues, aussi bien in vivo qu' in vitro. Cela est probablement dû : (1) à la faible sélectivité de plusieurs de ces agents ; (2) à l'importance de la libération à partir d'organites intracellulaires de $\mathrm{Ca}^{+}+$, cette libération n'étant pas bloquée par la plupart de ces agents, ou (3) au fait qu'une partie au moins de la libération de glu- tamate causée par l'anoxie n'est pas dépendante du $\mathrm{Ca}++$ extracellulaire [20, 21]. Par ailleurs, il est probable que l'implication de différents types de courants calciques dans de nombreuses fonctions essentielles à la survie rendra difficile le développement d'outils pharmacologiques ayant des effets uniquement anti-anoxiques.

- Le canal récepteur NMDA glutamate $[8,30]$

Étant donné l'importance de la libération excitotoxique de glutamate, de nombreuses études ont porté sur le

$$
\begin{gathered}
\text { Les canaux } \boldsymbol{K}+ \\
\text { dépendants de l'ATP } \\
(\boldsymbol{K}+-\boldsymbol{A T P}) \\
{[23,24,33,35-37]}
\end{gathered}
$$

Ces canaux ont pour propriété d'être inhibés par l'ATP intracellulaire, ils constituent par conséquent un véritable baromètre du métabolisme cellulaire puisqu'ils permettent d'établir un lien direct entre activité métabolique et excitabilité cellulaire. En effet, par l'intermédiaire d'une baisse de l'ATP intracellulaire, le stress métabolique permet l'activation de ces canaux et l'hyperpolarisation qui en résulte va réduire l'excitabilité cellulaire. Découverts initialement dans le muscle cardiaque [37], les canaux $K+$ dépendants de l'ATP ont été trouvés dans d'autres tissus tels les muscles lisses, les cellules $\beta$ pancréatiques et les cellules nerveuses [23, 35]. Leur rôle a été particulièrement bien étudié au niveau des cellules $\beta d u$ pancréas où ils contrôlent la libération d'insuline; une augmentation du glucose entraîne la fermeture de ces canaux (par l'intermédiaire de l'augmentation de l'ATP intracellulaire) induisant une dépolarisation suivie de l'activation de canaux $\mathrm{Ca}++$ dépendants du voltage et d'une libération d'insuline. L'hypoglycémie provoque un effet inverse. Plusieurs agents pharmacologiques ou hormones peuvent contrôler la libération d'insuline en agissant sélectivement sur ces canaux. Ainsi, les sulphonylurées, médicaments utilisés dans le traitement du diabète non insulinodépendant, libèrent l'insuline en bloquant ces canaux. En revanche, des activateurs de ces canaux comme le diazoxide provoquent une hyperglycémie par hypo-insulinémie. 
blocage des différents sites du canal récepteur NMDA. Ainsi le MK 801, qui bloque de façon irréversible le canal NMDA en se fixant dans le canal à l'état ouvert, bloque la lésion anoxique de CA1 in vivo et in vitro. Cet effet est observé même quand le MK 801 est administré plusieurs heures après le clampage des carotides, ce qui montre l'importance des effets tardifs de l'anoxie. L'APV un antagoniste sélectif du récepteur NMDA - réduit également la lésion anoxique. Il en est de même pour le 7-chloro-kynurénate - qui est un antagoniste sélectif du site allostérique du récepteur modulé par la glycine.

Sur le plan théorique, il est vraisemblable que, dans le centre du foyer nécrotique, on ne pourra pas empêcher des lésions; il est par conséquent utile de développer des agents pharmacologiques qui agissent, comme le MK 801, de façon postsynaptique sur les cellules de la région de pénombre afin de bloquer les effets toxiques de la libération excessive de glutamate par ces cellules nécrotiques. Par analogie avec le système GABA-benzodiazépine, il est possible que la modulation du site glycine s'avère, à long terme, plus efficace que le blocage du récepteur canal. Il reste cependant à voir si ces sites ne modulent pas la libération d'autres médiateurs - notamment inhibiteurs, leur blocage pouvant avoir dans ce cas des conséquences différentes de celles recherchées. Par ailleurs, la marge thérapeutique du MK 801 (qui a des effets secondaires importants) est très étroite et il est peu probable que l'on puisse administrer de façon préventive ce type d'agent. De plus, vu le décalage entre l'affinité du site glycine ( $\mathrm{Kd}<1 \mu \mathrm{M})$ et les taux de glycine endogène présumés $(>10 \mu \mathrm{M})$, il n'est pas évident que l'on puisse réduire de façon efficace les effets de l'anoxie en agissant sur le site modulateur glycinergique.

- Les récepteurs purinergiques [31, 32]

La présence de plusieurs types de récepteurs à l'adénosine a été décrite au niveau de l'hippocampe et d'autres structures nerveuses. Il existe notamment des récepteurs au

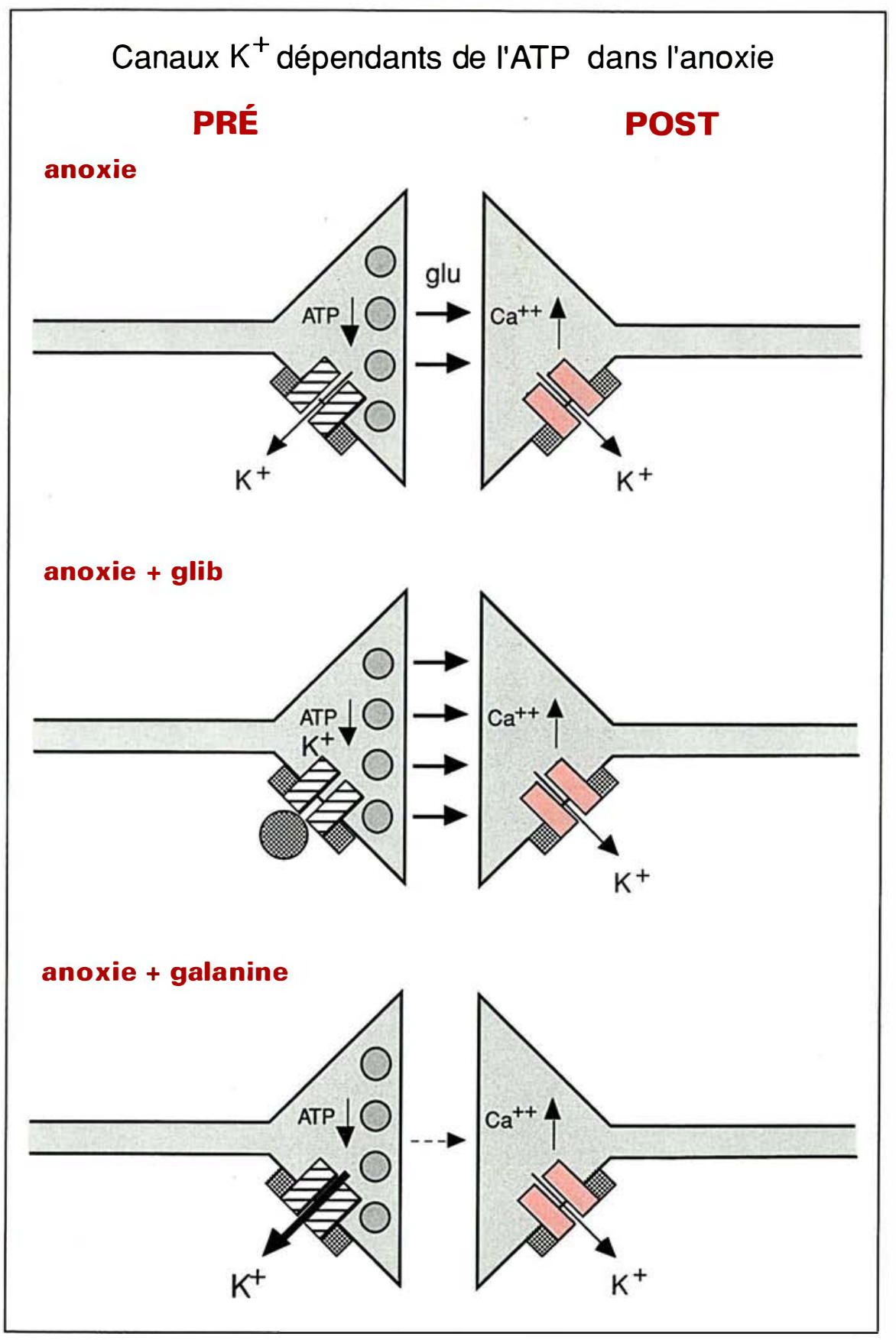

Figure 4. Effets de l'anoxie et rôle des canaux $K+$ au niveau des fibres moussues de I'hippocampe. L'anoxie produit une baisse d'ATP et une élévation du $\mathrm{Ca}^{+}+$intracellulaire au niveau des éléments pré-et post-synaptiques. La baisse d'ATP facilite l'activation des canaux $K^{+}$sensibles à l'ATP au niveau des terminaisons, la modulation de cet effet par la galanine ou la glibenclamide permet, respectivement, de réduire ou d'augmenter la libération de glutamate pendant l'anoxie. glu = glutamate. 
niveau des terminaisons collatérales de Schaffer qui innervent les cellules pyramidales de CA1; l'activation de ces récepteurs réduit la libération de médiateur (notamment de glutamate) par un mécanisme qui n'a pas été entièrement élucidé. Des antagonistes sélectifs de ces récepteurs protègent l'hippocampe des effets excitotoxiques de l'anoxie. Le mécanisme proposé est que, dans des conditions physiologiques, l'adénosine et d'autres molécules purinergiques sont libérées par les terminaisons en même temps que le glutamate ; ces molécules agissent en rétroaction sur les terminaisons afin d'empêcher une libération accrue de médiateurs dans l'anoxie. La libération excessive d'adénosine provoque une désensibilisation de ces récepteurs avec, comme conséquence, une perte de ce système régulateur et une libération non contrôlée de glutamate dans la phase post-anoxique. Il est probable que le développement d'une panoplie de drogues sélectives qui empêchent cette dérégulation ouvrira des perspectives intéressantes.

- Les canaux $\mathbf{K}+$ "ATP. dépendants " $[15,23,33]$

Les résultats que nous avons obtenus suggèrent que la modulation des canaux $\mathrm{K}+$ ATP-dépendants constitue une stratégie supplémentaire de contrôle de la libération de glutamate produite par l'anoxie (figure 4). Il est proposé que les récepteurs canaux $\mathrm{K}+$-ATP modulent la libération de transmetteurs dans des situations de stress métabolique, en particulier au niveau des fibres moussues de l'hippocampe, où il y a une densité relativement importante de récepteurs aux sulphonylurées [34]. Les agents qui modulent les canaux $\mathrm{K}+$-ATP pourraient avoir des propriétés antianoxiques efficaces: (1) car ces agents auront relativement peu d'effets dans des conditions d'oxygénation normales, puisque les canaux sont déjà bloqués par l'ATP ; (2) ils n'agiraient qu'au niveau de certaines structures cérébrales (si les récepteurs sont présents) et n'auraient pas d'effets relativement ubiquitaires comme d'autres molécules antianoxiques (canaux $\mathrm{Ca}++$ voltagedépendants, blocage des récepteurs glutamate ou NMDA). Dans un cadre plus général, il est intéressant de noter que plusieurs peptides, notamment la galanine ou la somatostatine, qui ouvrent ces canaux, sont libérés par des terminaisons parallèlement aux médiateurs conventionnels, dans certaines situations expérimentales de stress ou de stimulation excessive. Ces peptides auraient un rôle homéostatique, notamment en contrôlant la libération de médiateur par l'intermédiaire des canaux $\mathrm{K}+$ ATP-dépendants. La modulation des canaux $\mathrm{K}+$-ATP par différentes hormones - vraisemblablement par l'intermédiaire des protéines $\mathrm{G}$ - constitue un mécanisme de régulation qui va intervenir dans des situations de stress métabolique. Ces résultats ouvrent de nouvelles perspectives permettant d'envisager un traitement prophylactique de l'ischémie focale chez le sujet à risque, alors que les bloquants des récepteurs canaux NMDA seraient plutôt considérés comme un traitement d'urgence dans l'ischémie globale. De plus, la grande variété d'agents qui activent les canaux $\mathrm{K}+$ en général, et $\mathrm{K}+$-ATP en particulier, suggère qu'il devrait être possible de développer des outils pharmacologiques pourvus d'actions sélectives sur les canaux $\mathrm{K}+$-ATP de différentes structures cérébrales et périphériques [23]. Ainsi les canaux $\mathrm{K}+$-ATP du noyau ventro-médian de l'hypothalamus (qui semblent jouer un rôle important dans la régulation du comportement alimentaire), de même que ceux présents sur le muscle cardiaque, interviennent dans des situations d'hypoxie [35, 36].

Il est probable qu'aucune de ces stratégies ne suffise à couvrir le large champ des causes de séquelles neuronales des périodes d'anoxieischémie ; on peut raisonnablement penser, cependant, que le développement d'un spectre de molécules pourvues d'effets antianoxiques permettra de mieux appréhender le problème du traitement de l'anoxie chez l'homme

\section{Remerciements}

Je suis particulièrement reconnaissant à O. Robain et S. Zini pour leurs critiques du manuscrit et à $\mathrm{S}$. Bahurlet pour la frappe.

\section{Summary}

Ionic channels and anoxia

This paper reviews the effects of brief episodes of anoxia on synaptic transmission as well as the mechanisms of the lesions produced by repeated (or more sustained) anoxia. In hippocampal slices anoxia blocks synaptic transmission, the underlying mechanisms likely include a block of $\mathrm{Ca}++$ influx in the terminals caused by a rise in $(\mathrm{Ca})_{i}$ and $a$ concomitant fall of $(\mathrm{ATP})_{\mathrm{i}}$. Anoxia also produces a depolarization which is due to a delayed enhanced release of glutamate, this is thought to be a major cause in subsequent anoxic lesions (of the excitotoxic type). The various strategies to develop useful antianoxic agents are discussed. In particular, the potential usefulness of the recently described ATP sensitive $\mathrm{K}+$ channels which when open hyperpolarize the terminals and reduce transmitter release in the CNS is evaluated.

\section{TIRÉS A PART}

Y. Ben-Ari. 